\title{
Dried blood spots for Streptococcus pneumoniae and Haemophilus influenzae detection and serotyping among children $<5$ years old in rural Mozambique
}

Fabiana C. Pimenta ${ }^{1 *}$ (D), Benild Moiane ${ }^{2}$, Fernanda C. Lessa', Anne-Kathryn L. Venero³, laci Moura', Shanda Larson ${ }^{4}$, Sergio Massora², Alberto Chaúque ${ }^{2}$, Nelson Tembe², Helio Mucavele², Jennifer R. Verani', Cynthia G. Whitney', Betuel Sigaúque $^{2}$ and Maria G. S. Carvalho ${ }^{1}$

\begin{abstract}
Background: Dried blood spots (DBS) have been proposed as potentially tool for detecting invasive bacterial diseases.

Methods: We evaluated the use of DBS for S. pneumoniae and H. influenzae detection among children in Mozambique. Blood for DBS and nasopharyngeal (NP) swabs were collected from children with pneumonia and healthy aged $<5$ years. Bacterial detection and serotyping were performed by quantitative PCR (qPCR) (NP and DBS; lytA gene for pneumococcus and hpd for $\mathrm{H}$. influenzae) and culture (NP). Combined detection rates were compared between children with pneumonia and healthy.

Results: Of 325 children enrolled, 205 had pneumonia and 120 were healthy. Pneumococci were detected in DBS from 20.5 and $64.2 \%$ of children with pneumonia and healthy, respectively; NP specimens were positive for pneumococcus in 80.0 and $80.8 \%$, respectively. H. influenzae was detected in DBS from $22.9 \%$ of children with pneumonia and $59.2 \%$ of healthy; 81.4 and $81.5 \%$ of NP specimens were positive for $\mathrm{H}$. influenzae, respectively.

Conclusion: DBS detected pneumococcal and H. influenzae DNA in children with pneumonia and healthy. Healthy children were often DBS positive for both bacteria, suggesting that QPCR of DBS specimens does not differentiate disease from colonization and is therefore not a useful diagnostic tool for children.
\end{abstract}

Keywords: Dried blood spot, Pneumonia, Nasopharynx, Colonization, Streptococcus pneumoniae, Haemophilus influenzae

\footnotetext{
* Correspondence: gzy7@cdc.gov

${ }^{1}$ Division of Bacterial Diseases, National Center for Immunization and

Respiratory Diseases, Centers for Disease Control and Prevention, Atlanta

30329, USA

Full list of author information is available at the end of the article
}

(c) The Author(s). 2020 Open Access This article is licensed under a Creative Commons Attribution 4.0 International License, which permits use, sharing, adaptation, distribution and reproduction in any medium or format, as long as you give appropriate credit to the original author(s) and the source, provide a link to the Creative Commons licence, and indicate if changes were made. The images or other third party material in this article are included in the article's Creative Commons licence, unless indicated otherwise in a credit line to the material. If material is not included in the article's Creative Commons licence and your intended use is not permitted by statutory regulation or exceeds the permitted use, you will need to obtain permission directly from the copyright holder. To view a copy of this licence, visit http://creativecommons.org/licenses/by/4.0/. The Creative Commons Public Domain Dedication waiver (http://creativecommons.org/publicdomain/zero/1.0/) applies to the data made available in this article, unless otherwise stated in a credit line to the data. 


\section{Background}

Bacterial pneumonia is a leading cause of death in children worldwide, causing over 900,000 deaths annually in children aged < 5 years. The most common bacterial cause of pneumonia in children is $S$. pneumoniae, followed by $H$. influenzae type b (Hib) and Staphylococcus aureus $[1,2]$.

Since 1996, the Centro de Investigação em Saúde de Manhiça (CISM) has conducted surveillance for invasive bacterial diseases (IBD) among children in Mozambique rural area. Data from this surveillance system was instrumental for introducing the Hib vaccine in August 2009 [3] and 10-valent pneumococcal conjugate vaccine (PCV10) in April 2013. Invasive pneumococcal disease (IPD) incidence observed pre-PCV10 introduction was 245 cases per 100,000 among children < 5 years old, higher than what has been reported for this age group from other African sites, with an overall case fatality ratio of $14 \%$ [4].

Despite this high burden of IPD, the true incidence is likely underestimated given the challenges with IBD diagnosis. Diagnosis of IBD at CISM is currently made by culture; quantitative PCR (qPCR); and antigen tests (blood, cerebrospinal fluid or other sterile site fluids). Culture results can be highly influenced by prior antibiotic use, volume of specimen collected, specimen transport and storage conditions prior to processing, and culture media quality used to support the growth of fastidious bacteria like $S$. pneumoniae and $H$. influenzae [5]. To try to overcome these challenges, new potential diagnostic alternatives such as testing of dried blood spots (DBS) by qPCR have been proposed; yet they require validation before implementation [6-8].

DBS was first used for metabolic disorder screening in neonates, and its use was expanded to include pathogen detection [8]. DBS has been successfully used for detection of parasitic and viral diseases such as malaria, HIV, and dengue $[9,10]$. Testing DBS by qPCR is an attractive alternative to conventional diagnostic methods because the cards are low cost, require minimal blood volume, can be stored at room temperature, it is easy to collect enough blood via finger-prick, and testing for pathogens relies on deoxyribonucleic/ribonucleic acid (DNA/RNA) detection which may be less influenced by antimicrobial therapy than culture [9, 10]. However, little is known about their use as a diagnostic tool for detecting bacteria among children with IBD, and even less is known about whether qPCR testing of DBS will detect pathogens that are part of the normal flora in the upper respiratory tract of healthy children [10-12].

The development and validation of a diagnostic test that is not greatly impacted by prior antimicrobial use and that is both sensitive and specific would lead to a better understanding of the IBD burden, particularly in low- and mid-income countries where laboratory capacity is often limited. We examined detection of S. pneumoniae and $H$. influenzae by qPCR using DBS and the prevalence of nasopharyngeal colonization with these pathogens among children with pneumonia and healthy children $<5$ years from a rural area in Mozambique. For children with pneumonia who had undergone blood culture, we also compared those results with DBS findings.

\section{Methods}

\section{Study area and population}

The study was conducted between 2014 and 2015 among children aged < 5 years admitted to Manhiça District Hospital. Manhiça District is a rural area in southern Mozambique with a population of approximately 140,000 inhabitants. Since 1996, CISM has conducted IBD surveillance at this hospital, a 110-bed facility with 36 pediatric beds. Blood culture is routinely performed for all children aged $<2$ years on admission and for children aged 2-14 years admitted with axillary temperature $\geq 38^{\circ} \mathrm{C}$. Bacterial isolation and detection is performed using standard methods in the hospital laboratory [3, 4].

In order to evaluate the value of DBS test for distinguishing patients with pneumonia and potentially bacteremia from those without pneumonia and assess the influence of colonization on test results, we enrolled children with pneumonia and healthy children $<5$ years of age. Children with pneumonia were recruited at the hospital if they were hospitalized with severe pneumonia, while healthy children were randomly selected from the community using the Manhiça Demographic Surveillance System (DSS) [13]. Community workers visited household of selected healthy children for enrollment in the study. Severe pneumonia was defined as fever with cough or difficulty breathing associated with tachypnea and chest wall in-drawing [13]. Children recruited from the community were not enrolled if they had acute respiratory illness. The study protocol was approved by the Mozambique Ministry of Healthy and Centers for Disease Control and Prevention (CDC) Institutional Review Boards. Written informed consent was obtained from all parents or legal guardians prior to study enrollment. Demographic data were obtained for all participants.

\section{Specimen collection}

Blood and nasopharyngeal (NP) swabs were collected from each child by trained staff. Blood for DBS was collected first, followed by NP swabs. For children with pneumonia, specimen collection was performed within $48 \mathrm{~h}$ of admission. Blood was collected through finger or heel prick, depending on the child's age, and placed directly onto filter paper card containing 5 spots (Whatman Grade 903, cat\#10535097). After the blood dried, the card was put inside the aluminum card package, 
transported at room temperature to Manhiça laboratory, stored at $-20^{\circ} \mathrm{C}$, as an extra precaution to preserve DNA integrity, and shipped to the CDC Streptococcus Laboratory for processing.

A single NP swab was collected using a flexible and sterile calcium alginate tipped applicator (Puritan ${ }^{\circ} \mathrm{Cal}-$ giswab $^{\circ}$, cat\# 25-800 or 25-801). The swab was immediately placed in transport media containing $1.0 \mathrm{~mL}$ skim milk, tryptone, glucose, and glycerol (STGG-NP). Inoculated STGG-NP vials were kept at $4{ }^{\circ} \mathrm{C}$ within $4-5 \mathrm{~h}$ after collection and stored at $-70 \mathrm{C}^{\circ}$ until underwent culture and/or qPCR for S. pneumoniae and H. influenzae.

\section{Bacterial detection on DBS}

All the five spots from each DBS paper filter were cut into four pieces (approximately $250 \mathrm{uL}$ of blood), transferred to a round-bottomed tube, added $600 \mathrm{uL}$ of ATLbuffer (Qiagen cat\#1014758), and the tube vigorously vortexed for $10 \mathrm{~s}$. The solution was incubated at $85{ }^{\circ} \mathrm{C}$ for $10 \mathrm{~min}$, after added $20 \mathrm{uL}$ of proteinase-K $(600 \mathrm{mAU} /$ $\mathrm{mL}$ - Qiagen cat\#19133), vortexed for $10 \mathrm{~s}$, followed by incubation at $56{ }^{\circ} \mathrm{C}$ for $10 \mathrm{~min}$. A volume of $600 \mathrm{uL}$ of guanidinium-isothiocyanate-buffer (MagnaPure DNA Isolation Kit III - cat\#03264785001) was added to the sample and incubated at room temperature for $5 \mathrm{~min}$. The solution was transferred to the sample tube and DNA purification performed in the MagnaPure instrument set up for "DNA_Blood_external_lysis_V3_2". The DNA elution volume was 100uL.

DNA was stored at $-20^{\circ} \mathrm{C}$ until qPCR was performed using PerfeCTa ${ }^{\circ}$ qPCR ToughMix ${ }^{\oplus}$ Low ROX $^{\text {ma }}$ (cat\# 95114-012) for pneumococcal lytA gene [14], H. influenzae hpd gene [15]. hpd positive samples were serotyped by qPCR [16], and lytA positive samples were serotyped by quantitative multiplex PCR (qmPCR) assays covering the 37 most frequent pneumococcal serotypes $(1,2,3,4,5,6 \mathrm{~A} / 6 \mathrm{~B}, 6 \mathrm{C} / 6 \mathrm{D}, 7 \mathrm{~F} / 7 \mathrm{~A}, 9 \mathrm{~V} / 9 \mathrm{~A}, 11 \mathrm{~A} / 11 \mathrm{D}$, $12 \mathrm{~F} / 12 \mathrm{~A} / 12 \mathrm{~B} / 44 / 46,14,15 \mathrm{~F} / 15 \mathrm{~A}, 16 \mathrm{~F}, 18 \mathrm{~A} / 18 \mathrm{~B} / 18 \mathrm{C} /$ 18D, 19A, 19F, 22F/22A, 23A, 23F, 33F/33C/37) [17].

\section{S. pneumoniae and $H$. influenzae detection from NP swab specimens}

For pneumococcal isolation, a volume of $200 \mathrm{uL}$ of STGG-NP was transferred to $5.0 \mathrm{~mL}$ Todd Hewitt broth containing $0.5 \%$ yeast extract and $1.0 \mathrm{~mL}$ of rabbit serum; the broth was incubated at $35-37^{\circ} \mathrm{C}$ for $5 \mathrm{~h}$ and streaked onto blood agar plates for colony isolation [18]. Suspect colonies underwent optochin and bile solubility tests for pneumococcal identification. S. pneumoniae isolates were serotyped by Quellung reaction.

For $H$. influenzae isolation, a volume of $100 \mathrm{uL}$ of the STGG-NP was transferred to a chocolate agar plate with bacitracin and the plate incubated overnight in 5\% CO2 at $35-37^{\circ} \mathrm{C}$. Suspect colonies underwent Gram stain, oxidase, and X-V factor tests [19]. For species confirmation and serotyping, DNA was extracted from $H$. influenzae isolates and tested for $h p d$ and serotyping genes by qPCR $[15,16]$.

For NP specimens, DNA extracts were obtained from $200 \mathrm{uL}$ of STGG-NP using the protocol described above without the pretreatment step with ATL-buffer. DNA was stored at $-20^{\circ} \mathrm{C}$ until qPCR was performed for the pneumococcal lytA gene [14], H. influenzae hpd gene [15], and serotyping as described above [16, 17].

We compared the proportion of specimens that were positive for pneumococcus or $H$. influenzae among children with pneumonia and healthy using Chi-square or Fisher's Exact test when appropriate. Comparisons of DBS positivity among colonized and non-colonized children were also performed. $P$-values $<0.05$ considered statistically significant.

\section{Results}

Of 325 children enrolled, 205 had pneumonia and 120 were healthy controls from the community. Among the 203 and 119 children with pneumonia and healthy with age available, $134(66.0 \%)$ and $19(16.0 \%)$ were $\leq 1$ year of age, respectively. Pneumococcal lytA gene was detected from DBS in 20.5\% (42/205) of children with pneumonia and in $64.2 \%(77 / 120)$ of healthy. The $H$. influenzae hpd gene was detected from DBS in $22.9 \%$ (47/205) of children with pneumonia and in 59.2\% (71/ 120) of healthy. lytA and hpd genes were detected simultaneously from DBS in 13.2 and $46.7 \%$ of children with pneumonia and heathy, respectively (Table 1).

S. pneumoniae was detected in $80.0 \%(164 / 205)$ of children with pneumonia nasopharynx, 88.4\% (145/164) of it was isolated by culture (Table 1). Among healthy children, 80.8\% (97/120) were colonized with S. pneumoniae, $92.7 \%$ (90/97) of it was isolated by culture. Thirtyeight serotypes were identified amongst the pneumococcal isolates (Fig. 1). Five children with pneumonia were colonized with two pneumococcal serotypes. The lyt $\mathrm{A}$ gene was detected in 31.7\% (19/60) and 23.3\% (7/30) in NP swabs pneumococcal culture negative from children with pneumonia and healthy, respectively. Serotyping was performed on all 19 lyt A positive samples, but the serotype/serogroup was determined only for seven (3, 6A/6B, 18, 19A, 19F, 20, 23F). In children with pneumoniae $19.3 \%$ of S. pneumoniae isolates serotypes were included in the PCV10, (serotype 23F, $n=8$; 19F, $n=7$; 14, $n=5 ; 6 \mathrm{~B}, n=4 ; 9 \mathrm{~V}, n=2 ; 4, n=1 ; 18 \mathrm{C}, \mathrm{n}=1)$ and in healthy $32.2 \%$ (serotype $23 \mathrm{~F}, n=10$; $19 \mathrm{~F}, n=11$; $14, n=$ 3; 6B, $\mathrm{n}=4 ; 9 \mathrm{~V}, \mathrm{n}=1)$.

$H$. influenzae was detected in the nasopharynx of $81.4 \%(167 / 205)$ of children with pneumonia, $(116 / 167$ $69.4 \%$ ) isolated by culture (Table 1), most were nontypeable (NT) $(n=112)$, and four were serotyped 
Table 1 S. pneumoniae (lytA) and H. influenzae (hpd) detection from DBS and NP swabs collected from children with pneumonia and healthy children from a rural area in Mozambique, 2015

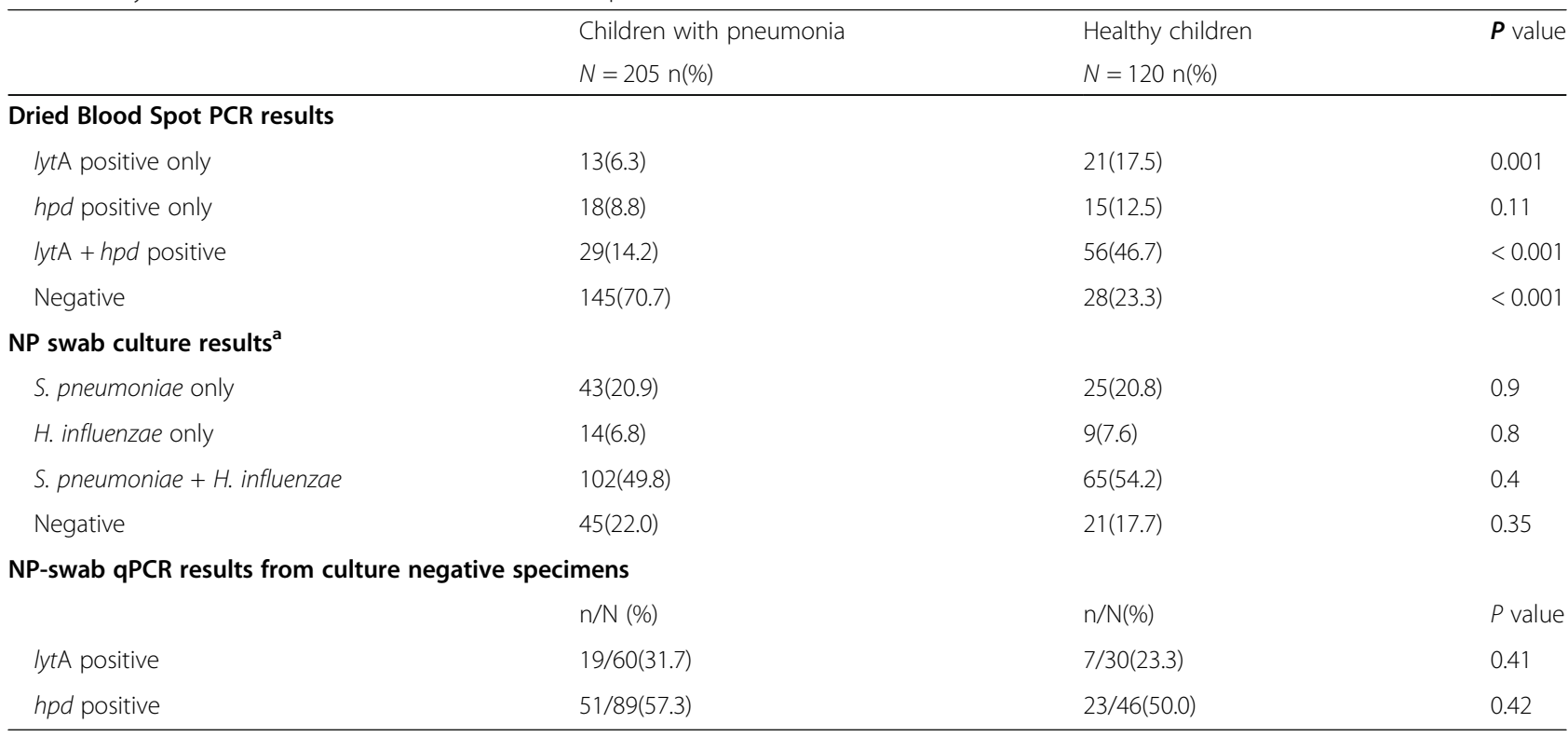

a $1(0.8 \%)$ NP swab was not processed for $H$. influenzae culture

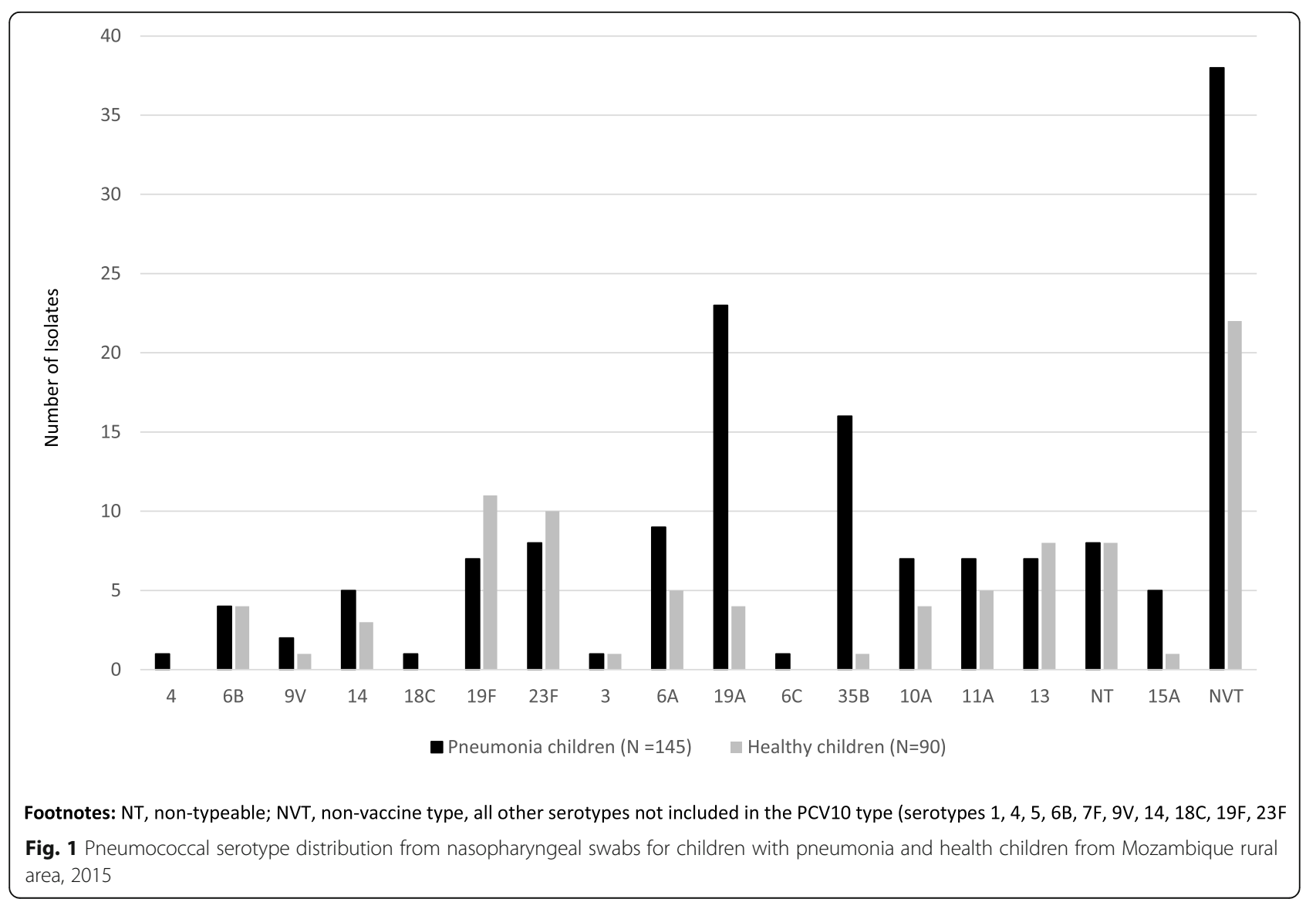


(serotype c, $n=1$; e, $n=3$ ). Among healthy children, 81.5\% (97/119) were colonized with $H$. influenzae in the nasopharynx; 76.2\% (74/97) isolated by culture, most were NT $(n=66)$ and eight were serotype (serotype a, $\mathrm{n}=3 ; \mathrm{b}, \mathrm{n}=3 ; \mathrm{c}, n=1 ; \mathrm{d}, \mathrm{n}=1)$. The hpd gene was detected in 57.3\% (51/89) NP swabs $H$. influenzae culture negative from children with pneumonia (NT, $n=45$; serotype a, $\mathrm{n}=1 ; \mathrm{b}, n=2$; e, $\mathrm{n}=2 ; \mathrm{f}, \mathrm{n}=1$ ) and in $51.1 \%$ $(23 / 45)$ among healthy children (NT, $n=16$; serotype a, $\mathrm{n}=2 ; \mathrm{b}, \mathrm{n}=1 ; \mathrm{c}, \mathrm{n}=1 ; \mathrm{e}, \mathrm{n}=2 ; \mathrm{f}, \mathrm{n}=2$ ). Co-colonization with S. pneumoniae and $H$. influenzae was detected in the nasopharynx of 111 (54.1\%) children with pneumonia and 69 (57.9\%) healthy.

The combination of pneumococcal colonization and detection of lytA in the DBS was found in $92.8 \%(39 / 42)$ of children with pneumonia, $85.7 \%(36 / 42)$ of the $S$. pneumoniae were isolated from the nasopharynx. While for healthy children this combination was found in $89.6 \%(69 / 77)$; $85.7 \%(66 / 77)$ of S. pneumoniae isolated by culture. The combination of $H$. influenzae colonization and hpd detection in DBS from children with pneumonia was found in $93.6 \%$ (44/47), for which $70.2 \%(33 / 47)$ of the $H$. influenzae were isolated from the nasopharynx; for healthy children it was $92.9 \%$ (66/ 71), 76\% (54/71) of $H$. influenzae isolated by culture (Table 1). DBS was more likely to be positive for pneumococci or $H$. influenzae if the subject was colonized, in both groups (Table 2).

Only DBS lytA positive specimens with DNA yield concentrations with cycle threshold value $(\mathrm{Ct}) \leq 32$ were able to be serotyped. Fifteen DBS lytA positive from children with pneumonia were serotyped; of these, $66.6 \%$ $(10 / 15)$ of the serotypes matched pneumococcal serotypes isolated in the nasopharynx. In healthy children, 30 DBS lytA positive were serotyped, and 33.3\% (10/30) were the same serotype/serogroup as was found in the nasopharynx. The pneumococcal serotype isolated in the nasopharynx of 16 children (7C, 10A, 13, 15B, 15C, 17F, 23B, 24F, 28F, 34, 35B, and 38) could not be compared with the DBS positive specimens because they are serotypes not included in the qmPCR assays.
Blood culture results were available for 185/205 (90.2\%) children with pneumonia: S. pneumoniae [6], Staphylococcus aureus [2], H. influenzae [1], Salmonella sp [1], Escherichia coli [1], and 174 were culture negative. All blood culture positive for $S$. pneumoniae were from children $\leq 1$ year old. The DBS and NP swabs from these six children were also positive for pneumococcus (100\% sensitivity). In three children, the same pneumococcal serotype was identified in the blood culture, DBS, and nasopharynx (serotype 6A $[n=2]$ and serotype 15A). We could not identify and compare the DBS pneumococcal serotype from the other three children because the $S$. pneumoniae isolated from the blood and nasopharynx were serotypes not encompassed in the qmPCR (10A, 13, 35B).

$H$. influenzae b was isolated in only one blood culture from a child $>1$ year old, and the same bacteria was also detected in the DBS and nasopharynx (100\% sensitivity). There were no differences in the proportion of children with pneumonia or healthy children who had a DBS positive test result or had pneumococcal or $H$. influenzae nasopharyngeal colonization by age group (Table 3 ).

\section{Discussion}

Our evaluation indicated that qPCR of blood from DBS specimens is not a useful method for distinguishing a bacterial pneumonia from bacterial colonization in children. DBS specimens were more likely to be positive for S. pneumoniae and/or $H$. influenzae when a child was colonized by these bacteria in the nasopharynx. We detected more pneumococcal and $H$. influenzae DNA in healthy children's DBS than in DBS samples from children with pneumonia, and co-detection of S. pneumoniae (lyt A) and H.influenzae (hpd) genes was found in almost half the DBS samples from healthy children. Bacterial DNA detection in blood from healthy children could be related to transient bacteremia or DNAemia from bacterial fragments in the bloodstream. Concerns have already been reported that positive pneumococcal detection in blood by qPCR may not reflect pneumococcal disease $[12,20,21]$. The usefulness of pneumococcal

Table 2 Results of bacterial detection from DBS specimens by NP colonization status among children with pneumonia and healthy children

\begin{tabular}{|c|c|c|c|c|c|c|c|}
\hline & \multicolumn{3}{|c|}{ With Pneumococcal colonization } & \multicolumn{3}{|c|}{ Without Pneumococcal colonization } & \multirow[t]{2}{*}{$P$ value } \\
\hline & Total & DBS positive & $\%$ DBS positive & Total & DBS Positive & $\%$ DBS positive & \\
\hline Children with pneumonia & 164 & 39 & 23.8 & 41 & 3 & 7.3 & 0.02 \\
\hline \multirow[t]{3}{*}{ Healthy children } & 97 & 69 & 71.1 & 23 & 8 & 34.8 & 0.001 \\
\hline & \multicolumn{3}{|c|}{ With $H$. influenzae colonization } & \multicolumn{3}{|c|}{ Without $H$. influenzae colonization } & $P$ value \\
\hline & Total & DBS Positive & $\%$ DBS positive & Total & DBS Positive & $\%$ DBS positive & \\
\hline Children with pneumonia & 167 & 44 & 26.3 & 38 & 3 & 7.9 & 0.02 \\
\hline Healthy children & 97 & 66 & 68.0 & 23 & 5 & 21.7 & $<0.001$ \\
\hline
\end{tabular}


Table 3 Proportion of DBS and NP specimens with S. pneumoniae and H. influenzae detected by age group* among children with pneumonia and healthy children

\begin{tabular}{|c|c|c|c|c|c|c|c|}
\hline & Age $\leq 1$ year & & & Age $>1$ to 5 years & & & $\boldsymbol{P}$ value \\
\hline & DBS positive & Total & $\%$ & DBS positive & Total & $\%$ & \\
\hline Children with pneumonia & 42 & 134 & 31.3 & 24 & 69 & 34.8 & 0.62 \\
\hline \multirow[t]{2}{*}{ Healthy children } & 14 & 19 & 73.7 & 80 & 100 & 80.0 & 0.53 \\
\hline & NP swab S.pneumoniae positive & Total & $\%$ & NP swab S.pneumoniae positive & Total & $\%$ & \\
\hline Children with pneumonia & 112 & 134 & 83.6 & 51 & 69 & 73.9 & 0.10 \\
\hline \multirow[t]{2}{*}{ Healthy children } & 16 & 19 & 84.2 & 81 & 100 & 81.0 & 0.74 \\
\hline & NP swab H.influenzae positive & Total & $\%$ & NP swab H.influenzae positive & Total & $\%$ & \\
\hline Children with pneumonia & 108 & 134 & 80.6 & 58 & 69 & 84.0 & 0.54 \\
\hline Healthy children & 17 & 19 & 89.5 & 80 & 100 & 80.0 & 0.33 \\
\hline
\end{tabular}

*age missing for 2 children with pneumonia and 1 healthy child

qPCR on blood was reported as limited in diagnosing childhood pneumococcal pneumonia by the Pneumonia Etiology Research for Child Health (PERCH) since positivity among controls (5.5\%) was comparable to that in pneumoniae cases not confirmed for any bacterial pathogens $(6.3 \%)$, and among cases confirmed for nonpneumococcal bacteria (11.2\%) [21].

Limited data are available for bacterial detection using DBS and many studies have not included healthy controls when evaluating the performance of DBS for detection of pneumococcus or $H$. influenzae. A study with Nigerian children only found $0.96 \%$ positivity for $S$. pneumoniae on DBS of 1038 febrile children and 1.3\% S. pneumoniae positive from 79 healthy children [22].

Before DBS collection began, we performed tests to optimize methods using serial dilution of human blood spiked with S. pneumoniae, S. agalactiae and S. pyogenes isolates from several serotypes to evaluate the Whatman Grade 903 (cat\#10535097), and the FTA Elute Micro CardTM (cat\#WB120401) (data not shown). Several protocols for DBS DNA extraction were also tested [11, 23, 24], including some automated extraction systems (Nuclisens EasyMag and MagNAPure). Only after extensive testing of these protocols a final protocol with the most optimal results was established for this study (data not shown).

Each DBS spot has a $12 \mathrm{~mm}$ diameter, corresponding to approximately $50 \mathrm{uL}$ of blood. Previously reported protocols had DNA extracted from just one DBS spot $(50 \mathrm{uL})$ or one $3 \mathrm{~mm}$ punch containing around $12 \mathrm{uL}$ of blood [11, 12, 23-25]. The relatively low volume of blood extracted, and DNA added into the GPCR reaction is likely to be the major factor for the lack of sensitivity and inconsistent bacterial detection in previous DBS testing. The improved DNA extraction protocol allowed for use of all five $(250 \mathrm{uL})$ blood spots on each card. This increase yielded higher availability of purified bacterial DNA, which in association with a more stable Taq
DNA polymerase that better resisted common residual qPCR inhibitors from blood, allowed for better performance of the amplification reaction. The methods applied here enhanced DBS qPCR testing for S. pneumoniae detection from 2 to $9 \%$ positive (1 spot from the DBS card) in previous reports $[12,26]$ to $20.5 \%$ (5 spots) when testing symptomatic children. While these methods were not helpful for diagnosis of disease caused by pneumococci or $H$. influenzae, the technique might be useful for detection of systemic infections caused by bacteria that are not commonly carried in the upper respiratory tract.

Pneumococcal nasopharyngeal carriage prevalence, considering the detection by culture and qPCR, was high for children with pneumonia (80\%) and for those who were healthy $(80.8 \%)$, like what has been previously reported in cross-sectional pneumococcal carriage surveys conducted in the same rural area in Mozambique [27, 28].

Children who were colonized with S. pneumoniae or $H$. influenzae were at least 2-fold more likely to have a DBS positive for the pathogen they were carrying compared to those who were not colonized.

Our results agree with Morpeth et al. [21] that reported higher blood pneumococcal qPCR positivity (almost 2-fold) among those controls with nasopharyngeal carriage. The impact of colonization on DBS test results limits the specificity of DBS for detecting disease, particularly in areas where colonization is common. The high bacterial load in the nasopharynx often found in young children could have an impact on the amount of DNA in the child's bloodstream and urine. Similarly, tests for detecting pneumococcal antigen in urine were significantly more likely to be positive among children who were nasopharyngeal carriers of pneumococci than for those who were not [29]. The increased likelihood of qPCR detection in blood samples when testing children with densely colonized nasopharynx was also reported [30]. The accuracy of pneumococcal detection by qPCR 
in serum has also been evaluated for children who had pneumococci detected by culture of cerebrospinal fluid and blood; qPCR detected pneumococci in serum from these children, but serum was also positive in $17 \%$ of healthy controls [20].

The use of DBS for of S. pneumoniae and H. influen$z a e$ detection was previously evaluated on children hospitalized with pneumonia from Mozambique and Morocco [26]. Even though our study was conducted in the same District in Mozambique, our results differ. The detection rates for pneumococci and $H$. influenzae among children with pneumonia were 9.0 and $3.3 \%$ in the earlier study compared to 20.5 and $22.9 \%$ in our study, respectively. Among healthy children, these authors [26] found only $1.9 \%$ were DBS positive for pneumococci compared to $64.2 \%$ in ours. They also could not associate pneumococci isolated from blood culture $(3.1 \%)$ with the corresponding DBS, whereas in our study the same serotypes of $S$. pneumoniae and $H$. influenzae found on blood culture (3.2\%) were simultaneously identified in the DBS and NP swabs of children with pneumonia. A few factors could explain the differences: 1) our results are based on a comprehensive population study with paired nasopharyngeal and DBS results from children with pneumonia and healthy; 2) all children enrolled in our analysis were $<5$ years old, but Selva et al. [26] enrolled children up to 10 years old (older children have lower colonization rates); 3) we optimized DNA extraction procedure that allowed all five DBS spots from each card to be included, resulting in approximately $250 \mathrm{uL}$ of blood volume extracted but Selva et al. [26] used $100 \mathrm{uL}$; 4) we used a different nucleic acid purification system; and 5) we also used a Taq DNA polymerase that is more resistant to qPCR inhibitors from blood.

Our study has several limitations. First, we restricted enrollment of children with pneumonia to one hospital, Manhiça District Hospital, which is the main referral hospital for the District from where healthy children were recruited. As a referral hospital, it admits many patients after they have been treatment elsewhere. We also could not assess the time of administration of inpatient antimicrobial use in relation to the collection of DBS. Some enrolled children likely received the intravenous antibiotics prior to collection of DBS. Collection of specimens from pneumonia children after they received antibiotics, including any antibiotics received before admission as well, may have reduced detection of pneumococcal DNA from their specimens. A crosssectional survey conducted in the same period and area of Mozambique found $97.4 \%$ of children with pneumonia had antibiotics recently compared to $27 \%$ among children without pneumonia [27]. The vaccination status of the children was obtained, and Mozambique has high vaccine coverage [28]. However, we could not assess the date of the last PCV10 dose compared to the swab or blood collection. Manhiça has a high pneumococcal colonization rate, similar to other countries in Africa, but different from developed countries [28, 31]. DBS may perform better in settings where pneumococcal colonization is not as prevalent as in Mozambique.

Another limitation is that in several instances we could not determine the serotype from lytA positive DBS samples, either because the DNA concentration recovered was too low $(\mathrm{Ct}>32)$ or the serotypes were not encompassed in the qmPCR assays.

\section{Conclusions}

We found that DBS positive results were highly associated with pneumococcal and $H$. influenzae nasopharyngeal colonization, suggesting that qPCR testing for DBS samples did not distinguish colonization from invasive disease and therefore is not likely to be useful for diagnosis of pneumonia etiology in children.

\section{Abbreviations \\ CDC: Centers for Disease Control and Prevention; CISM: Centro de Investigação em Saúde de Manhiça; DBS: Dried Blood Spots; DSS: Demographic Surveillance System; IBD: invasive bacterial diseases; IPD: invasive pneumococcal disease; NP: nasopharyngeal; NT: non-typeable PERCH: Pneumonia Etiology Research for Child Health; PCV10: 10-valent pneumococcal conjugate vaccine; qmPCR: quantitative multiplex PCR; qPCR: quantitative PCR; STGG: skim milk, tryptone, glucose, and glycerol; STGG-NP: skim milk, tryptone, glucose, glycerol inoculated with nasopharyngeal swab}

\section{Acknowledgements}

The authors thank the children and their parents for participating in the study.

\section{Authors' contributions}

All authors have read and approved the manuscript. FCP designed the study, participated in testing validation, analysis, interpretation of data, had drafted the original work, and substantively revised it; BM participated and managed the acquisition of specimens, data curation, and testing; FL designed the study, participated in funding acquisition, analysis, interpretation of data, and substantively revised it; AKV participated in testing and analysis; IM participated in testing and analysis; SL participated in testing and analysis; SM participated in acquisition of specimens; AC managed acquisition of specimens, data curation, and analysis; NT participated in the analysis; HM participated in the analysis, data curation; JRV designed the study, analysis, and substantively revised it; CW designed the study, and substantively revised it; BS designed the study, analysis, and revised it; MGSC designed the study, participated in funding acquisition, testing validation, analysis, supervision, interpretation of data, and substantively revised it.

\section{Funding}

US Federal Government Funds supported laboratory supplies, facilities and personnel for this study.

\section{Availability of data and materials}

The data generated or analyzed during this study are included in this published article.

\section{Ethics approval and consent to participate}

The study protocol was approved by the Mozambique Ministry of Healthy and Centers for Disease Control and Prevention (CDC) Institutional Review Boards. Written informed consent was obtained from all parents or legal guardians prior to study enrollment. 


\section{Consent for publication}

Not applicable.

\section{Competing interests}

The authors declare that they have no competing interests.

The findings and conclusions in this report are those of the authors and do not necessarily represent the official position of the Centers for Disease Control and Prevention.

\section{Author details}

'Division of Bacterial Diseases, National Center for Immunization and Respiratory Diseases, Centers for Disease Control and Prevention, Atlanta 30329, USA. ${ }^{2}$ Centro de Investigação em Saúde de Manhiça, 1929 Maputo, Mozambique. ${ }^{3}$ IHRC Inc., Atlanta 30346, USA. ${ }^{4}$ Weems Design Studio Inc., Suwanee 30024, USA.

Received: 20 March 2020 Accepted: 16 June 2020 Published online: 02 July 2020

\section{References}

1. O'Brien KL, Baggett HC, Brooks WA, Feikin DR, Hammitt LL, Higdon MM, et al. Causes of severe pneumonia requiring hospital admission in children without HIV infection from Africa and Asia: the PERCH multi-country casecontrol study. Lancet. 2019;394(10200):757-79.

2. WHO. Pneumonia Fact Sheet 2016. http://www.who.int/mediacentre/ factsheets/fs331/en/. Accessed 19 July 2019.

3. Sigaúque B, Roca A, Mandomando I, Morais L, Quintó L, Sacarlal J, et al. Community-acquired bacteremia among children admitted to a rural hospital in Mozambique. Ped Inf Dis J. 2009;28:108-13.

4. Sigaúque B, Verani JR, Acácio S, Mandomando I, Bassat Q, Nhampossa TL, et al. Burden of invasive pneumococcal disease among children in rural Mozambique: 2001-2012. PLOS One. 2018;13:e0190687.

5. Wilson ML, Weinstein MP, Reller B. Laboratory detection of bacteremia and fungemia. In: Carroll K, Pfaller M, Landry M, McAdam A, Patel R, Richter S, Warnock D, editors. Manual of clinical microbiology. 12th ed. Washington: ASM Press; 2019. p. 28-44.

6. Peters RP, van Agtmael MA, Danner SA, Savelkoul PH, VandenbrouckeGrauls CM. New developments in the diagnosis of bloodstream infections. Lancet Infect Dis. 2004;4:751-60.

7. Meesters RJW, Hooff GP. State-of-the-art dried blood spot analysis: an overview of recent advances and future trends. Bioanalysis. 2013;5:2187208.

8. Enderle $Y$, Foerster $\mathrm{K}$, Burhenne J. Clinical feasibility of dried blood spots: analytics, validation, and applications. J Pharm Bio. 2016;130:231-43.

9. Koontz D, Baecher K, Amin M, Nikolova S, Gallagher M, Dollard S. Evaluation of DNA extraction methods for the detection of cytomegalovirus in dried blood spots. J Clin Virol. 2015;66:95-9.

10. Wihokhoen B, Dondorp AM, Turner P, Woodrow CJ, Imwong M. Use of blood smears and dried blood spots for polymerase chain reaction-based detection and quantification of bacterial infection and Plasmodium falciparum in severely ill febrile African children. Am J Trop Med Hyg. 2016; 94:322-6.

11. Selva $L$, Krauel $X$, Pallares $R$, Munoz-Almagro C. Easy diagnosis of invasive pneumococcal disease. Emerg Infect Dis. 2011;17:1125-7.

12. Iroh Tam PY, Hernandez-Alvarado N, Schleiss MR, Hassan-Hanga F, Onuchukwu C, Umoru D, et al. Molecular detection of Streptococcus pneumoniae on dried blood spots from febrile Nigerian children compared to culture. PLOS One. 2016:11:e0152253.

13. Acácio S, Verani JR, Lanaspa M, Fairlie TA, Nhampossa T, Ruperez M, et al. Under treatment of pneumonia among children under 5 years of age in a malaria-endemic area: population-based surveillance study conducted in Manhica district- rural, Mozambique. Int J Infect Dis. 2015;36:39-45.

14. Carvalho Mda G, Tondella ML, McCaustland K, Weidlich L, McGee L, Mayer $L W$, et al. Evaluation and improvement of real-time PCR assays targeting lytA, ply, and psaA genes for detection of pneumococcal DNA. J Clin Microbiol. 2007:45:2460-6.

15. Wang X, Mair R, Hatcher C, Theodore MJ, Edmond K, Wu HM, et al. Detection of bacterial pathogens in Mongolia meningitis surveillance with a new real-time PCR assay to detect Haemophilus influenzae. Int J Med Microbiol. 2011;301:303-9.
16. WHO Manual. Laboratory Methods for Diagnosis of Meningitis Caused by Neisseria meningitides, Streptococcus pneumoniae, Haemophilus influenzae. 2nd ed. 2011. p.32-258.

17. Pimenta FC, Roundtree A, Soysal A, Bakir M, du Plessis M, Wolter N, et al. Sequential triplex real-time PCR assay for detecting 21 pneumococcal capsular serotypes that account for a high global disease burden. J Clin Microbiol. 2013;51:647-52.

18. da Gloria CM, Pimenta FC, Jackson D, Roundtree A, Ahmad Y, Millar EV, et al. Revisiting pneumococcal carriage by use of broth enrichment and PCR techniques for enhanced detection of carriage and serotypes. J Clin Microbiol. 2010:48:1611-8.

19. Brandileone MC, Zanella RC, Almeida SCG, Brandao AP, Ribeiro AF, Carvalhanas TMP, et al. Effect of 10-valent pneumococcal conjugate vaccine on nasopharyngeal carriage of Streptococcus pneumoniae and Haemophilus influenzae among children in São Paulo, Brazil. Vaccine. 2016;34:5604-11.

20. Dagan R, Shriker O, Hazan I, Leibovitz E, Greenberg D, Schlaeffer F, et al. Prospective study to determine clinical relevance of detection of pneumococcal DNA in sera of children by PCR. J Clin Microbiol. 1998;36: 669-73.

21. Morpeth SC, Deloria Knoll M, Scott JAG, Park DE, Watson NL, Baggett HC, et al. Detection of pneumococcal DNA in blood by polymerase chain reaction for diagnosing pneumococcal pneumonia in young children from low- and middle-income countries. Clin Infect Dis. 2017;64:S347-56.

22. Iroh Tam PY, Hernandez-Alvarado N, Schleiss MR, Yi AJ, Hassan-Hanga F, Onuchukwu C, et al. Detection of Streptococcus pneumoniae from culturenegative dried blood spots by real-time PCR in Nigerian children with acute febrile illness. BMC Res Notes. 2018;11:657-66.

23. de Vries JJ, Claas EC, Kroes AC, Vossen AC. Evaluation of DNA extraction methods for dried blood spots in the diagnosis of congenital cytomegalovirus infection. J Clin Virol. 2009;4:S37-42.

24. MacKenzie CR, Henrich B. Methods in molecular biology. In: de Vries JJ, Barbi M, Binda S, editors. Claas ECJ editors. New York: Springer Science Business Media; 2012. p. 169-75.

25. Smit PW, Elliott I, Peeling RW, Mabey D, Newton PN. An overview of the clinical use of filter paper in the diagnosis of tropical diseases. Am J Trop Med Hyg. 2014;90:195-210.

26. Selva L, Benmessaoud R, Lanaspa M, Jroundi I, Moraleda C, Acacio S, et al. Detection of Streptococcus pneumoniae and Haemophilus influenzae type b by real-time PCR from dried blood spot samples among children with pneumonia: a useful approach for developing countries. PLOS One. 2013; 8(10):e76970.

27. Adebanjo T, Lessa FC, Mucavele H, Moiane B, Chauque A, Pimenta F, et al. Pneumococcal carriage and serotype distribution among children with and without pneumonia in Mozambique, 2014-2016. PLOS One. 2018;13(6): e0199363.

28. Sigaúque B, Moiane B, Massora S, Pimenta F, Verani JR, Mucavele $H$, et al. Early declines in vaccine type pneumococcal carriage in children less than 5 years old after introduction of 10-valent pneumococcal conjugate vaccine in Mozambique. Ped Infect Dis J. 2018;37(10):1054-60.

29. Dowell SF, Garman RL, Liu G, Levine OS, Yang YH. Evaluation of Binax NOW an assay for the detection of pneumococcal antigen in urine samples, performed among pediatric patients. Clin Infect Dis. 2001;32:824-5.

30. Isaacman DJ, Zhang Y, Reynolds EA, Ehrlich GD. Accuracy of a polymerase chain reaction-based assay for detection of pneumococcal bacteremia in children. Pediatrics. 1998;101:813-6.

31. Althouse BM, Hammitt LL, Grant L, Wagner BG, Reid R, Larzelere-Hinton F, et al. Identifying transmission routes of Streptococcus pneumoniae and sources of acquisitions in high transmission communities. Epidemiol Infect. 2017;145:2750-8.

\section{Publisher's Note}

Springer Nature remains neutral with regard to jurisdictional claims in published maps and institutional affiliations. 